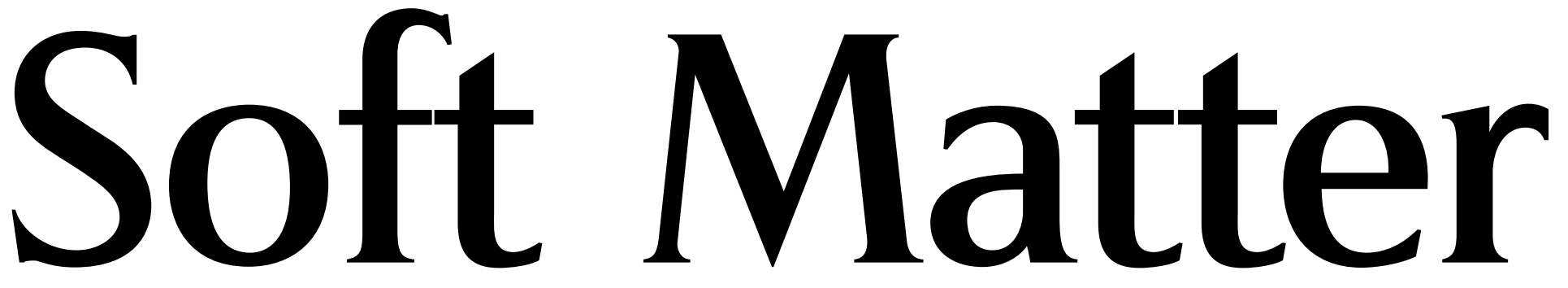

\section{PAPER}

Ramon Pericet-Camara et al.

Solid-supported thin elastomer films

deformed by microdrops

\section{HIGHLIGHT}

Jianzhong Du and Rachel K. O'Reilly

Advances and challenges in smart and

functional polymer vesicles 


\title{
Solid-supported thin elastomer films deformed by microdrops $\dagger$
}

\author{
Ramon Pericet-Camara, ${ }^{* a b}$ Günter K. Auernhammer, ${ }^{b}$ Kaloian Koynov, ${ }^{b}$ Simone Lorenzoni, ${ }^{c}$ Roberto Raiteri ${ }^{c}$ \\ and Elmar Bonaccurso $* b$
}

\author{
Received 8th April 2009, Accepted 24th June 2009 \\ First published as an Advance Article on the web 30th July 2009 \\ DOI: 10.1039/b907212h
}

\begin{abstract}
A sessile droplet can deform the surface of a soft solid not only with its weight. The surface tension pulls up a ridge at the perimeter of the drop, and the capillary pressure embosses a quasi-spherical dimple underneath the drop. This holds for the case of a bulk solid. However, if the solid forms a film with thickness comparable to the deformation scale the shape and the depth of the dimple are strongly distorted. We investigated dimples on elastomer films with a Young's modulus of $25 \mathrm{kPa}$ and thickness in the range 4-104 $\mu \mathrm{m}$ embossed by sessile ionic liquid droplets. The films are supported by an undeformable glass slide. Below a certain critical film thickness, the dimple is shallower and the ridge at the drop rim is less elevated than for the bulk elastomer. The deviations are more pronounced for thinner films. Further, troughs form at the two sides of the ridge. Their distance from the rim is equivalent to the layer thickness. The measurements are qualitatively reproduced by an analytical model and quantitatively by numerical simulations. A consistent physical picture of the deformation on the bulk elastomer and of the distortions on the thin films is given.
\end{abstract}

\section{Introduction}

The study of wetting of a solid surface by a liquid is of high interest not only from a fundamental point of view, but it is relevant in numerous industrial applications as diverse as the spreading of paints and coatings on surfaces, the lubrication of bearings, crop spraying, surface cleaning, or printing processes. ${ }^{1}$ The physical heterogeneity of a surface (random roughness or controlled topology) and its chemical heterogeneity (surface energy) play a key role in wetting processes, as already demonstrated by Wenzel $^{2}$ and $\mathrm{Cassie}^{3}$ and confirmed by an increasing number of investigations on static and dynamic wetting ${ }^{4-7}$ since then. The influence of a third surface property on wetting, on the other hand, has been studied to a much smaller extent: its 'softness' or 'elasticity'. In fact, a droplet of liquid of millimeter size resting on a sufficiently soft solid material causes a warping of the surface. This deformation is not due to the weight of the droplet, but solely to its surface tension. The exact shape of the deformation has been calculated by several authors. ${ }^{8-10}$ In early work, it was merely predicted that a ridge of height $h$ is formed at the three-phase contact line (TPCL - rim of the drop) due to the surface tension of the liquid $\gamma_{\mathrm{L}}$ pulling upwards. In later work, also the contribution of the capillary or Laplace pressure $\Delta P$ was taken into consideration. A dimple of depth $d$ was predicted to form under the area wetted by the drop (Fig. 1a). The presence of the ridge outside the droplet had been measured optically by

aiocolloid and Fluid Physics Group, Department of Applied Physics, University of Granada, Campus Fuentenueva, Av. Severo Ochoa s/n, 18071 Granada, Spain.E-mail: rpericet@ugr.es

${ }^{b}$ Max Planck Institute for Polymer Research, Ackermannweg 10, 55128 Mainz, Germany.E-mail: bonaccur@mpip-mainz.mpg.de

'Department of Biophysical and Electronic Engineering, University of Genova, Via Opera Pia 11a, 16145 Genova, Italy

$\dagger$ Electronic supplementary information (ESI) available: Rationalization of the formation of the microtrough in terms of minimization of a simplified free energy expression. See DOI: 10.1039/b907212h
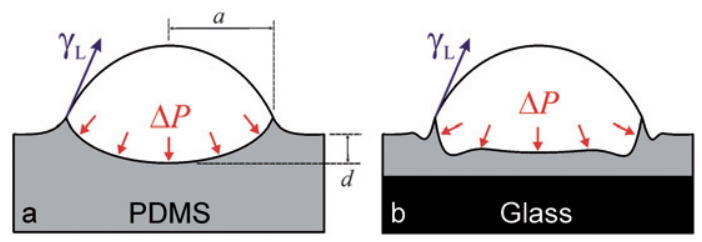

Fig. 1 Schematic of the deformation on (a) bulk soft surfaces and (b) thin soft films due to the action of surface tension $\gamma_{\mathrm{L}}$ and capillary pressure $\Delta P$. The drop radius is $a$ and the dimple depth $d$.

several authors. ${ }^{11-14}$ Only recently, however, Pericet-Camara et $a .^{15}$ characterized and analyzed the entire deformation of a soft Sylgard ${ }^{\circledR} 184$ surface by a sessile microdrop by means of laser scanning confocal microscopy (LSCM). Sylgard is an elastomer mainly consisting of poly(dimethylsiloxane) (PDMS). The elastic modulus $E$ was tuned between 25 and $340 \mathrm{kPa}$. Ridges and dimples several microns high or deep produced by the surface tension on the microdrop were found. The measurements were all made on thick films since the theoretical models were developed for semi-infinitely thick solids. Finite film thickness and the influence of a solid support underneath the film were addressed in two papers, by Fredrickson et al. ${ }^{16}$ and Long et al. ${ }^{17}$ The first one dealt with the wetting of nanometer-thick films of polymer brushes, while the second treated a generic elastic film. Both sets of authors described the formation of the ridge only by the surface tension pulling perpendicularly upwards along the wetting front of a liquid film. Their models predict a symmetric profile on the two sides of the ridge, of the form of an exponentially decaying sine wave. The periodicity of the sine is similar to the film thickness. More recently, also finite element simulations of the deformation of solid-supported elastic films by droplets have been reported. ${ }^{18}$ The elastic modulus was in the range $360-740 \mathrm{kPa}$ and the layer thickness $t$ was between 0.5 and $10 \mathrm{~mm}$. The contact radius of the drop was $a=1.16 \mathrm{~mm}$. The 
authors considered the effect of the capillary pressure and could predict the formation of a dimple underneath the drop. They found that its depth decreased with decreasing film thickness, and that the profile was not symmetric on the two sides of the TPCL. For the thinnest $(t=0.5 \mathrm{~mm})$ and softest $(E=360 \mathrm{kPa})$ film, their results show a weak undulation of the profile on the inside of the drop. On the outside, the spatial resolution of the simulations was too low to allow visualizing the undulation. The authors, however, did not address this feature in their analysis. Summarizing the works cited above, one can expect that a droplet resting on a thin elastic film will generate a ridge and a dimple with an oscillating but non-symmetric profile on the two sides of the TPCL (Fig. 1b). To the best of our knowledge, such a system has not been directly characterized and modelled yet.

The quantitative prediction of such a deformation is relevant for the modelling of static, but foremost dynamic wetting processes on thin elastic films, like polymer coatings, car paintings, or the coating of printing plates with inks. In a series of experiments, Shanahan and Carré ${ }^{19,20}$ demonstrated that the wetting dynamics of a liquid on a surface is slower for softer substrates. They called the phenomenon 'viscoelastic braking' and explained it as follows: the liquid front spreading on a soft surface deforms the surface due to capillary forces. In order to further spread, the contact line has to overcome an additional energy needed for deforming the surface. The energy dissipation due to the viscoelastic deformation of the surface reduces the spreading speed. ${ }^{11}$ The authors compared the ideal spreading velocity on a hard and undeformable surface and a soft and deformable surface. They analyzed the profile of the deformation generated by the advancing liquid front and developed a semiquantitative model. Applying this model to thick films and bulk elastomers, Voué et al. ${ }^{21}$ found that viscoelastic breaking dissipates additional energy during liquid spreading for films with $t>30 \mu \mathrm{m}$. When the film thickness was reduced below $3 \mu \mathrm{m}$, liquid spreading was governed by the viscous dissipation inside the drop only. These works stressed the influence of elasticity and of film thickness on dynamic wetting, but still no quantitative information on the extent of the deformation of the thin film was obtained. This is the aim of the present work. We optically measured deformation profiles inside and outside of sessile droplets resting on soft films of different thickness and fixed elastic modulus $E=25 \mathrm{kPa}$. We compared the results with the analytic models by the groups of Fredrickson ${ }^{16}$ and Long ${ }^{17}$ and found good quantitative agreement with results from finite element simulations. In the ESI, $\uparrow$ we rationalize the formation of the microtrough in terms of minimization of the simplified free energy expression derived by Frederickson.

\section{Experimental}

\section{Materials}

We used sessile drops of the ionic liquid 1-butyl-3-methylimidazolium hexafluorophosphate (Fluka, Switzerland) doped with the fluorophore Nile Red (Sigma-Aldrich, Germany) at $1 \mathrm{mM}$ concentration. The surface tension of the ionic liquid is $\gamma_{\mathrm{L}}=48.8 \mathrm{mN} / \mathrm{m}^{22}$ and the viscosity at room temperature is $\eta=312 \mathrm{mPa} \cdot \mathrm{s}^{23}$
The thin elastic films are made of Sylgard ${ }^{\circledR} 184$ (Dow Corning, USA), a commercial two-component silicone elastomer based on PDMS. The base (monomer) is mixed with a curing agent (crosslinker). The amount of curing agent determines the elastic modulus of the film. We use a ratio of $50: 1$ by weight of the base/curing agent to produce Sylgard with an elastic modulus $E=25 \mathrm{kPa}$, as measured by tensile tests. The Sylgard film behaves purely elastically in the range of applied strains. The nominal Poisson's ratio of the film is $\nu=0.5$.

\section{Fabrication of thin films}

Thin round glass slides (microscope cover slips, MENZEL, Germany) with a diameter of $25 \mathrm{~mm}$ and thickness of $\sim 0.15 \mathrm{~mm}$ are used as solid supports for the soft films. Uncrosslinked Sylgard is spin-coated onto the slides. Afterwards, the samples are cured for $1 \mathrm{~h}$ in an oven at $80^{\circ} \mathrm{C}$. The thickness of the films is controlled by the rotation speed of the glass slide and by the overall spin-coating time. Thicknesses between 4 and $104 \mu \mathrm{m}$ are obtained by spinning at speeds from 500 to $10000 \mathrm{rpm}$ for $120 \mathrm{~s}$, at an initial acceleration of $500 \mathrm{rpm} / \mathrm{s}$ and a final deceleration of $20 \mathrm{rpm} / \mathrm{s}$. The thickness of the films is measured by scratching the Sylgard with a needle at various positions and imaging the samples with a $\mu \operatorname{surf}^{\circledR}$ white-light confocal profilometer (Nanofocus AG, Germany). The thickness of the Sylgard is homogeneous over the whole glass slide, with deviations of less than $1 \%$ from the mean thickness. The surface topography of ten Sylgard samples was analyzed. The average slope of the studied regions with scan size of $160 \mu \mathrm{m} \times 160 \mu \mathrm{m}$ was $0.6 \%$, and it was never above $1 \%$. After correction of this tilting, a mean peak-to-peak value of $R_{\max }=35 \pm 7 \mathrm{~nm}$ was found. This value includes the waviness of the surface. The average surface roughness of the Sylgard was $R_{\mathrm{a}}=2.7 \pm 0.5 \mathrm{~nm}$.

\section{Ink-jet deposition of microdrops}

Ionic liquid droplets are deposited on the films with an ink-jet dispenser (Nano-Plotter NP 2.0, GeSiM mbH, Germany). A pipette heatable up to $125^{\circ} \mathrm{C}$ is used to warm up the viscous ionic liquid before dispensing it. Droplets of contact radii between 60 and $300 \mu \mathrm{m}$ are deposited at different positions of the same sample to form a regular array.

\section{Imaging of microdrops and deformed surface}

We used a commercial laser scanning confocal microscope (LSCM) setup (Carl Zeiss, Jena, Germany) consisting of the module LSM 510 and an inverted microscope model Axiovert 200. In all experiments, a $40 \times$ Plan-Neofluar multi-immersion objective (Carl Zeiss, Jena, Germany) with numerical aperture $\mathrm{NA}=0.9$ and oil as the immersion liquid was used. The excitation light source was a He-Ne laser operating at a wavelength $\lambda=543 \mathrm{~nm}$. In order to image simultaneously the PDMS surface and the ionic liquid droplet, two detection channels were used. The first one was configured in reflection mode, i.e. a BP505-550 emission filter was applied to detect the excitation laser light reflected from the PDMS/air interface. The second channel was configured to detect the fluorescence of the Nile Red dispersed in the ionic liquid using an LP560 emission filter (Fig. 2). By moving the focus plane in the $Z$-direction, single images showing optical 

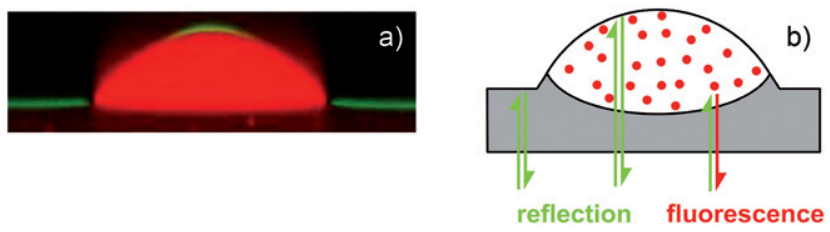

Fig. 2 (a) Experimental image of an ionic liquid droplet labelled with fluorescent Nile Red on a soft PDMS surface obtained with laser scanning confocal microscopy (LSCM). The green line shows the reflected light at the solid-vapour interface. (b) Schematic representation of a droplet containing a fluorescent dye on an elastic surface scanned by LSCM. The green arrows symbolize the exciting laser and the reflection on the solid-vapour interface, and the red arrow indicates the emitted fluorescence.

slices of the system can be put together to build up a $3 \mathrm{D}$ reconstruction of the fluorescing ionic liquid droplet on the reflecting elastic surface. ${ }^{15}$

The fluorescence image of the droplet is thresholded with the Isodata algorithm ${ }^{24}$ implemented in ImageJ analysis software (Wayne Rasband, National Institute of Health, USA). In this way, the location of the interfaces of the droplet with the elastic surface and with air can be precisely determined. The intensity of the reflected light at the solid-vapour interface has a Gaussian distribution when scanned perpendicular to the surface. Only the centre of that distribution is displayed in the graphs below and represents the exact position of the interface. In order to further increase the resolution close to the TPCL on the outside of the drop, we acquire additional images with the $\mu$ surf $^{\circledR}$ white-light confocal profilometer and combine them with the reflection data from the LSCM measurements. The tilting of the Sylgard surfaces is corrected by levelling the obtained images with the white-light confocal profilometer to the horizontal. However, this correction was not possible for the images acquired with LSCM, and they keep the surface slope.

\section{Simulations}

The numerical (based on finite elements - FE) simulations were performed using commercial software (COMSOL Multiphysics 3.5 equipped with the Thermal-Structural Interaction package). In the range of deformations discussed here, the PDMS film can be safely regarded as a linear elastic and incompressible material. Hence, Poisson's ratio was set to 0.5 . The radius of the PDMS film considered in the model was always three times the radius of the ionic liquid droplets, in order to respect the condition of semiinfinite layer. The drop radius could vary from 5 to $300 \mu \mathrm{m}$, while the PDMS thickness ranged from below $10 \mu \mathrm{m}$ to above $100 \mu \mathrm{m}$. The mesh for the PDMS film consisted of about 30000 tetrahedral elements (the number of elements varied according to the thickness of the film) and, consequently, of about 120000 degrees of freedom. A finer mesh size for the elements close to the TPCL was used in order to better resolve the stress caused by the droplet at its rim. The boundary conditions were set as follows: the nodes on the axis of symmetry of the PDMS film cannot move in the radial direction; moreover, the nodes on the bottom of the film cannot move in any direction because the PDMS film is considered to fully adhere to a rigid substrate. The capillary pressure generates a compressive stress over the wetted area which is uniformly distributed over all nodes of the contact area. The vertical component of liquid surface tension generates a tensile stress at the rim of the drop which is uniformly distributed over all nodes at the contact line. The deformation of the drop was not taken into consideration.

\section{Results and discussion}

We monitored the deformation of the solid-liquid-vapour interface between sessile ionic liquid drops and soft elastomers of varying thickness with elastic modulus $E=25 \mathrm{kPa}$. We acquired 3D images with LSCM and plotted cross-sections through the drops perpendicularly to the surface at the drop diameters (Fig. 3). The red area is the fluorescence of the ionic liquid drop and the green line is the reflection from the solid-vapour interface. No reflection is detected at the solid-liquid interface due to the similar refractive indexes of ionic liquid and elastomer (around $n=1.43$ for a wavelength of $\lambda=633 \mathrm{~nm}$ ). We extracted the profile inside the drop from the fluorescence signal and outside the drop from the reflection signal. We thus obtained a map of the entire deformation of the surface. In Fig. 3a, the image of a droplet (red area) with $a=60 \mu \mathrm{m}$ on a film with thickness $t=104 \mu \mathrm{m}$ is shown. Deformation of the surface due to gravity is negligible for such small drops. On the other hand, the capillary pressure embosses a parabolic dimple $\sim 2.8 \mu \mathrm{m}$ deep underneath the wetted area. ${ }^{15}$ As well, a ridge $\sim 0.7 \mu \mathrm{m}$ high is formed at the TPCL due to the non-balanced vertical component of the surface tension of the drop. We additionally acquired

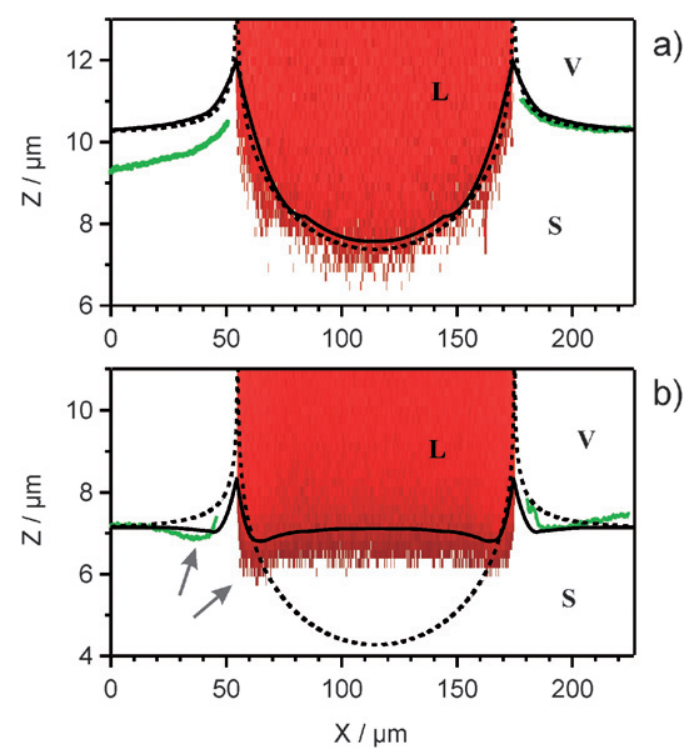

Fig. 3 LSCM images of an ionic liquid sessile droplet of $a=60 \mu \mathrm{m}$ contact radius on an elastomer surface of elastic modulus $E=25 \mathrm{kPa}$ and Poisson's ratio $\nu=0.5$. The red area indicates the fluorescing droplet. The green line indicates the solid-vapour interface. The black dashed line is calculated according to the model of Rusanov, ${ }^{9}$ and the solid black line is the simulated profile. The contact angle of the drop with the surface is $\theta=74^{\circ}$ and the surface tension of the ionic liquid is $\gamma_{\mathrm{L}}=48.8 \mathrm{mN} / \mathrm{m}$. The grey arrows point the microtroughs at the two sides of the TPCL (see text for details). S, V and L indicate the solid (Sylgard), vapour (air), and liquid (microdrop) regions, respectively. Thickness of the elastomer layers are: (a) $t=104 \mu \mathrm{m}$ and (b) $t=8.5 \mu \mathrm{m}$. 
surface profiles on the outer side of the drop with a white-light profilometer. These profiles are comparable to those acquired by LSCM, but the $Z$-scale resolution is enhanced. For comparison, theoretical profiles are calculated with the continuum elastic model first presented by Rusanov ${ }^{9}$ (dashed line) and with FE-simulations (solid line). The substrate is considered as a semiinfinite and homogeneous elastic solid. The experimental profile (green line) agrees well with the calculated profiles within the experimental error of the LSCM measurement. The discrepancy in the left part of the image between the calculated and the measured profiles is due to a slight tilting of the sample with respect to the horizontal. The experimental deformation profile shown in Fig. $3 \mathrm{~b}$ is for a similar drop with $a=60 \mu \mathrm{m}$, but resting on a much thinner film with thickness $t=8.5 \mu \mathrm{m}$.

\section{Deformation due to the capillary pressure}

The profile under the drop on the thin film is different from that on the thick film: the dimple is shallower (depth $\approx 0.7 \mu \mathrm{m}$ ), the ridge is lower (height $\approx 0.2 \mu \mathrm{m}$ ), but foremost the dimple under the drop is not of parabolic shape. It is flat, even slightly convex in the central area, and on both sides of the TPCL shallow microtroughs are formed. Similar microtroughs have been observed also upon micropatterning thin polymer films ${ }^{25}$ and bulk polymer surfaces ${ }^{26-28}$ by microdrops of solvents, and also upon micropatterning bulk polymer surfaces by the uptake of solvent vapour using non-solvent sessile droplets as shadow masks. ${ }^{29}$

The characteristic shape of the profile is a direct effect of the finite film thickness and of the incompressibility of the film material. ${ }^{16,17}$ Sylgard has Poisson's ratio $\nu=0.5$ and is thus incompressible. The deformation is axisymmetric, like for the bulk case. The measured deformation profile (green line and bottom border of red area) is well reproduced by the finite element simulation (solid line). The difference is in the range of the experimental error of the LSCM measurements. We will discuss and comment on the FE-simulation results in more detail in the last paragraph.

To quantify the influence of film thickness $t$ on deformation, we plotted the depths $d$ of the dimples measured at the centre of the drops versus $t$ (Fig. 4). The film thickness $t$ varied between

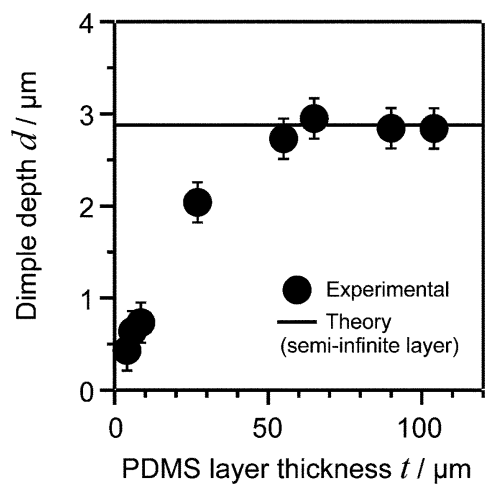

Fig. 4 Dimple depth $d$ under the drop as a function of the layer thickness $t$. The error bars correspond to the step between two subsequent optical slices. The solid line is the limiting value $d_{\text {Rus }}$ for semi-infinite solids as calculated from Rusanov's model. ${ }^{9}$
4 and $104 \mu \mathrm{m}$. The contact radius of the drop $a$ was $60 \mu \mathrm{m}$ and the elastic modulus of the film $E$ was $25 \mathrm{kPa}$. The dimple depth for a semi-infinitely thick layer of Sylgard (limiting value $t_{\text {lim }}$ ) was calculated from Rusanov's model and plotted for comparison (solid line). For film thickness $t>55 \mu \mathrm{m}$ the experimental and the theoretical depths coincide. When $t<55 \mu \mathrm{m}$ the depth of the dimple becomes progressively smaller. We cannot assume any further that the film thickness is semi-infinite. As the film becomes thinner, the solid support increasingly exerts an influence on the deformation.

The phenomenon is similar to that observed in works of nanoindentation, ${ }^{30}$ where such a solid-supported film has been termed a 'mechanical double-layer'. ${ }^{31}$ In our system the sessile drop 'indents' the soft surface exerting a downwards-oriented force via its capillary pressure. The effect is very similar to the pressure exerted by a hard tip indenting a substrate. According to literature, the effect of the solid support under the soft film cannot be neglected when the indentation is deeper than around $10 \%$ of the film thickness. ${ }^{32} \mathrm{We}$ found a similar value here. We fitted a line to the measured depths for films up to $30 \mu \mathrm{m}$ thickness and extrapolated it to larger thicknesses. This line crossed the horizontal line of the limiting value calculated according to Rusanov's model $\left(d_{\mathrm{Rus}}=2.9 \mu \mathrm{m}\right)$ at $t_{\mathrm{lim}}=36.7 \mu \mathrm{m}$. Beyond this film thickness, the influence of the solid support could be neglected. The depth of the dimple is $8 \%$ of the limiting film thickness, which is very close to the limiting value of $10 \%$ measured in and calculated for indentation measurements.

\section{Deformation due to the surface tension}

Besides the capillary pressure, the surface tension of a sessile drop also exerts an upwards directed force at the TPCL. The consequence in our system is the formation of a ridge at the rim of the drop. Depending on the contact angle between drop and surface and on the elasticity of the surface, the height of the ridge can be up to several microns (see Fig. 3). For a semi-infinite film, the profile of the solid-vapour interface outside the drop shows a logarithmic decay ${ }^{9,19}$ and it is in good agreement with the profile calculated with Rusanov's model. For films of finite thickness, the profile is better described by a decaying and oscillating exponential. As for the profile inside the drop, only the first minimum can be measured and forms a microtrough just next to the TPCL. Finite element simulations of the outer part of the profiles agree well with the measured profiles.

The resolution of the LSCM profiles in proximity of the TPCL is rather poor. This technique can only collect the light reflected by surfaces which are not tilted beyond a certain threshold angle. For our instrument, this angle is around $5^{\circ}$. We thus used a white-light confocal profilometer to acquire the 3D drop profiles in reflection mode. The threshold angle of our instrument is around $35^{\circ}$ so that steeper surfaces can be measured. Also, the vertical resolution is in the nanometer range and allows for an increased $Z$-scale resolution.

Fig. 5 shows the high resolution measured profiles close to the TPCL outside of drops with similar contact radii of $a=60 \mu \mathrm{m}$. The thickness of the Sylgard film varies between 4 and $104 \mu \mathrm{m}$. The calculated deformation profile for a semi-infinite layer is plotted for comparison (dashed line). The profile for a $104 \mu \mathrm{m}$ thick film is well reproduced by Rusanov's model. 


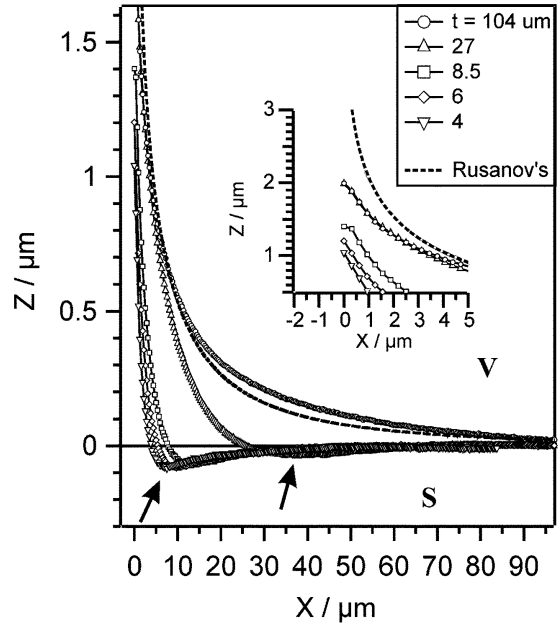

Fig. 5 Profiles of the solid-vapour interface of a Sylgard film $(E=25 \mathrm{kPa}$ and $\nu=0.5)$ warped by an ionic liquid droplet $(a=60 \mu \mathrm{m}$, $\theta=74^{\circ}$ and $\gamma_{\mathrm{L}}=48.8 \mathrm{mN} / \mathrm{m}$ ). The different symbols of the profiles correspond to different thicknesses $t$ of the Sylgard film. The inset shows the profiles close to the TPCL. The two arrows point to the microtroughs (see text for details). S stands for the solid (Sylgard) and V for the vapour (air) phase.

A slight deviation is found only in close proximity (within $5 \mu \mathrm{m}$ ) of the TPCL. This means that the solid support does not influence either the height or the shape of the ridge. On the other hand, as expected, the deformation profile progressively deviates from the semi-infinite bulk model as the layer thickness $t$ is reduced below the limiting thickness $t_{\mathrm{lim}}=36.7 \mu \mathrm{m}$. Further, the profiles of films of thickness $t$ from 27 down to $4 \mu \mathrm{m}$ do not show a monotonic logarithmic decay: microtroughs are formed before the profiles level off again. They are concentric with the TPCL, up to $100 \mathrm{~nm}$ deep and up to $50 \mu \mathrm{m}$ broad. Microtroughs are deeper, narrower and closer to the TPCL for thinner Sylgard films.

A zoom of the profiles next to the TPCL is shown in the inset of Fig. 5. We set the highest measured point at $X=0$, but this choice has to be taken with caution. The imaging technique cannot resolve the complete ridge because the slope of the upper part is at an angle higher than $35^{\circ}$. A simple extrapolation of the profiles indicates that the TPCL is $1-2 \mu \mathrm{m}$ beyond the first measured data point. The measured maximum heights decrease with decreasing film thickness: the ridge formed on the $4 \mu \mathrm{m}$-thick film is around $50 \%$ smaller than on the $104 \mu \mathrm{m}$ (semiinfinite) film. This trend and the shape of the profile can be qualitatively reproduced by the $1 \mathrm{D}$ model developed by Long et al. ${ }^{17}$ for the static wetting of thin soft rubber films by a liquid. In this model, the free surface deformation $\Delta h(x)$ is calculated explicitly. The contribution of the capillary pressure is neglected and the surface tension is modelled as a punctual force pulling vertically upwards at the TPCL. The deformation is limited by the free energy increase due to the expansion of the film free surface and the elastic bulk deformation. Through the minimization of the total energy, the equilibrium deformation is calculated along $x$ in three regions with qualitatively different behaviours (see also the ESI $\dagger$ for an illustrative explanation):

(1) At distances from the TPCL larger than the film thickness $(|x|>t)$ :

$$
\Delta h(x) \propto \frac{-\gamma_{\mathrm{L}} \sin \theta}{E} \sin \left(\frac{|x|}{\xi_{1}}\right) \exp \left(\frac{-|x|}{\xi_{2}}\right)
$$

where $\xi_{1}$ and $\xi_{2}$ are characteristic lengths of the order of the film thickness $t$.

(2) At intermediate distances $\gamma_{\mathrm{L}} / E<|x|<t$ :

$$
\Delta h(x) \cong \frac{-\gamma_{\mathrm{L}} \sin \theta}{2 \pi E} \ln \left(\frac{|x|}{t}\right)
$$

(3) At short distances $|x|<\gamma_{\mathrm{L}} / E$ the deformation is limited to a finite value:

$$
\Delta h(x) \cong \frac{-\gamma_{\mathrm{L}} \sin \theta}{2 \pi E} \ln \left(\frac{\gamma}{E t}\right)
$$

The profile of the deformation is an oscillating decaying exponential, symmetric on both sides of the TPCL. We have compared calculations based on Long's model with two examples of our experimental data (Fig. 6). The model was developed for a film thickness $t$ much smaller than the drop contact radius $a$. We thus chose two films, with $t_{1}=4 \mu \mathrm{m}$ (Fig. 6a) and $t_{2}=8.5 \mu \mathrm{m}$ (Fig. 6b), and with $a=60 \mu \mathrm{m}$. Surface tension $\gamma_{\mathrm{L}}$, Young's modulus $E$ and film thickness $t$ are fixed in the model; $\xi_{1}$ and $\xi_{2}$ have been put equal to the film thickness $t$. The agreement between model and experiment is qualitatively good

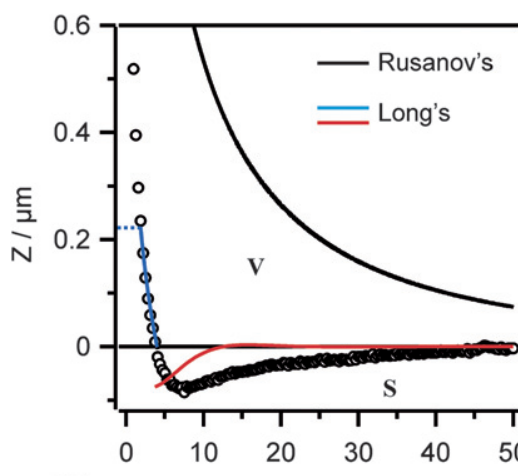

a)

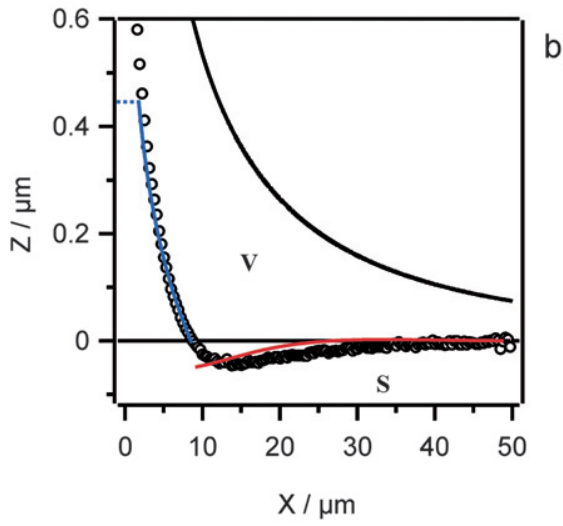

Fig. 6 Profile of the solid-vapour interface close to the TPCL of an ionic liquid droplet of contact radius $a=60 \mu \mathrm{m}$ on an elastomer surface with $E=25 \mathrm{kPa}$ Young's modulus. The symbols are the experimental profiles. The coloured lines represent Long's model. The blue and red lines are respectively close and far from the contact line. The TPCL region is shown as a dotted blue line. The black line is Rusanov's model. Elastomer layer thickness is (a) $t=4 \mu \mathrm{m}$, and (b) $t=8.5 \mu \mathrm{m}$. 
in both cases. The model predicts the formation of a first trough at a distance from the TPCL approximately equal to the film thickness. We could confirm this feature with our measurements (Fig. 5 and Fig. 6). We could not measure further oscillations beyond the first. However, calculations with the material parameters we used predict the second oscillation to be only a few nanometers in amplitude, which is beyond the resolution of our technique. The model predicts well the depth and position of the trough, and the profile close to the ridge (blue line), but less well the profile far from the ridge (red line). We plotted eqn (1.1) (red line) using a proportionality constant $A_{1}=-0.12$ (Fig. 6a) and $A_{2}=-0.08$ (Fig. 6b). Eqn (1.2) is displayed as a blue line in the interval established by the model. The lower limit, $x=\gamma_{\mathrm{L}} / E$, marks the start of the TPCL region, whose height value $\Delta h$ is calculated from eqn (1.3). The model predicts ridge heights lower than the experimental ones, but it has clear limitations: expressions (1.1)-(1.3) are only close approximations to the exact solution. Additionally, they are for much thinner films than the drop size. Further, the deformation caused by the capillary pressure is not included.

We plotted the distance $x_{0}$ of the microtroughs from the TPCL $(X=0)$ on the outside of the drop as a function of film thickness $t$ (Fig. 7). The model by Long et al ${ }^{17}$ predicts a nearly $1: 1$ relation between both lengths and is plotted as a solid line. For thin films up to $t=10 \mu \mathrm{m}$, the model exactly matches the measured distances (inset of Fig. 7). For thicker layers, the agreement is weaker, but still satisfactory considering the simplicity of the model. The scattering of the data for a given film thickness is due to using different drops. Thus, the distance $x_{0}$ appears to be a constant of the system.

FE-simulations of the deformation produced by sessile microdrops on soft Sylgard films are compared to the experimental deformation profiles of the solid-vapour surface and to Rusanov's model (Fig. 3 and Fig. 8). The theoretical models predict that the ridge height increases with increasing drop radius and decreases with decreasing film thickness. The dimple depth is independent of drop size for semi-infinitely thick films, and decreases with film thickness for $t<t_{\text {lim }}$. To demonstrate the

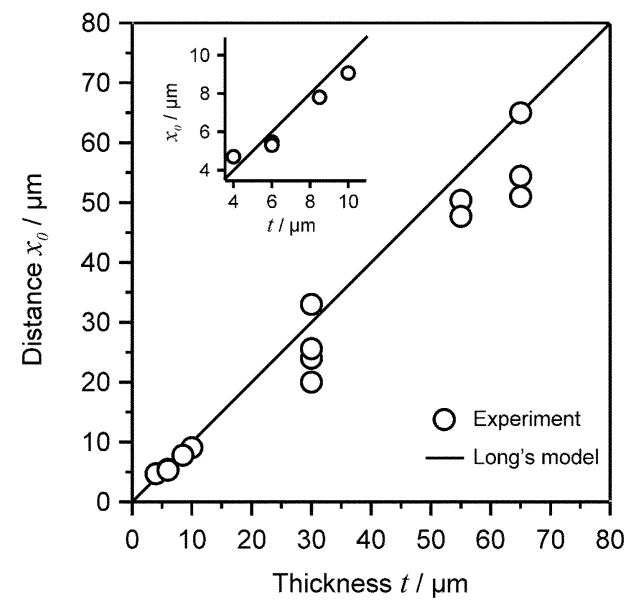

Fig. 7 Distance $x_{0}$ of the microtrough from the TPCL as a function of the Sylgard film thickness $t$. The circles are the experimental data and the line is the prediction from Long's model. ${ }^{17}$ The inset shows a zoom-in for thin films.
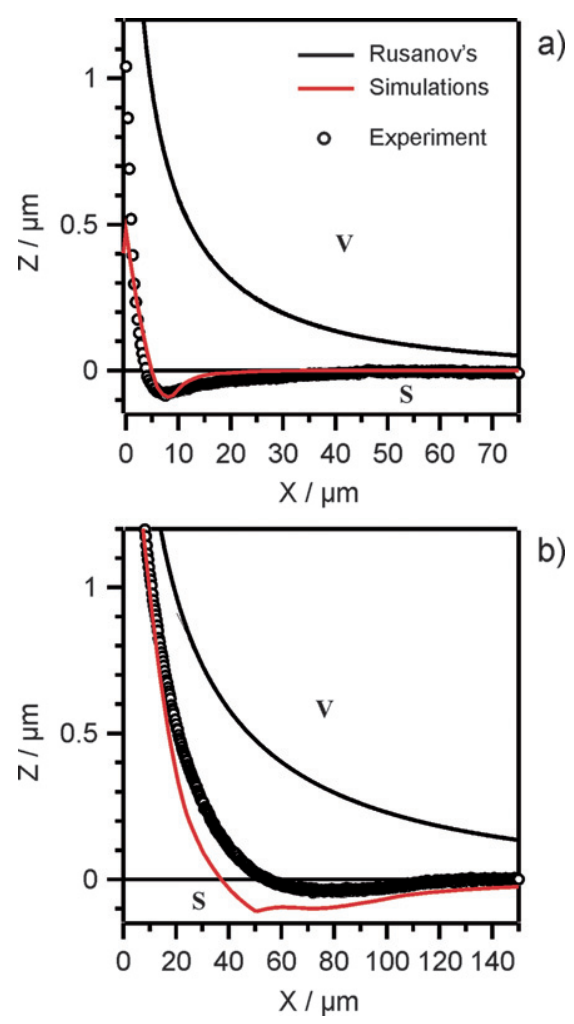

Fig. 8 Lateral profile of the solid-vapour interface close to the TPCL of an ionic liquid droplet on an elastomer surface of $25 \mathrm{kPa}$ Young's modulus. The symbols are the experimental profiles. The red lines are the simulation profiles of the systems. The black lines are Rusanov's model. S and V mark the solid (elastomer) and the vapour (air) regions. (a) Droplet of radius $a=60 \mu \mathrm{m}$ and elastomer layer thickness $t=4 \mu \mathrm{m}$, and (b) $a=266 \mu \mathrm{m}$ and $t=60 \mu \mathrm{m}$.

predictive power of the simulations, we present two extreme cases, though all simulations match similarly well with the measurements: droplets with contact radii of 60 and $266 \mu \mathrm{m}$ on films with thicknesses of 4 and $60 \mu \mathrm{m}$. The upper profile (Fig. 8a) is relatively steep close to the TPCL, the microtrough begins at a distance $x_{0}=4 \mu \mathrm{m}$ from the TPCL, is around $80 \mathrm{~nm}$ deep, and levels off at a distance of around $50 \mu \mathrm{m}$ from the TPCL. The experimental deformation is, as expected, in full disagreement with Rusanov's model, but in good agreement with the finite element simulation. The simulated ridge is lower and the microtrough only slightly narrower than the measured ones. In comparison, the ridge of the lower profile (Fig. 8b) is higher and the profile is less steep in the proximity of the TPCL. The microtrough begins at $x_{0}=54 \mu \mathrm{m}$ from the TPCL, is only around $40 \mathrm{~nm}$ deep, but extends up to around $150 \mu \mathrm{m}$ where it levels off. Agreement with profiles calculated according to the Long model is fair as well. Also, the simulation reproduces the main features of the experimental profile, such as the ridge height and slope, and the general trend with one microtrough and no further measurable oscillations. Yet, there is a slight difference concerning the position and the depth of the microtrough. A number of factors could explain this slight discrepancy. The $X-Y$ resolution of the imaging technique is $300 \mathrm{~nm}$, while the $Z$ resolution is around $5 \mathrm{~nm}$ in this case. For such shallow features as the ones measured here, even a slight tilting of the sample can influence 
the accuracy of the measured values, especially since they are close to the native waviness of the Sylgard surface of around $10 \mathrm{~nm}$.

\section{Conclusions}

The effect of film thickness on its deformation caused by a nonevaporating and immobile sessile droplet has been studied. Both the dimple formed under the drop due to the capillary pressure and the ridge formed at the rim of the drop due to the surface tension are affected by the solid support underneath the soft elastic thin film. For a film with an elastic modulus of $25 \mathrm{kPa}$, we found that the hard substrate influences the depth of the dimple when the deformation is above $8 \%$ of the film thickness. These results agree with indentation measurements on similar thin films, where a threshold value of around $10 \%$ is found. Below such a film thickness, the profiles of the dimple and the ridge do not monotonically decrease any more, but show exponentially decaying oscillations. In particular, our measurements revealed only the first oscillation, i.e. a minimum in the form of a microtrough. The distance of the microtrough from the rim of the drop is a constant of the system and is approximately equivalent to the film thickness. In fact, the free energy of the system exhibits a clear minimum for Fourier components of the surface profile in the order of the inverse layer thickness. This favours Fourier modes close to the minimum and tries to reduce Fourier modes far away from it. As argued in the ESI, $\dagger$ balancing these two tendences leads to the formation of a minimum in the deformation profile at distances from the TPCL in the order of the layer thickness. This conclusion is also important when it comes to wetting studies on deformable surfaces, because it can provide a mechanism for tuning the wetting dynamics via controlling film elasticity and thickness. This mechanism could be implemented, for instance, in microfluidic devices. We thus see the results of this work as a starting point for investigating the fundamental process of dynamic wetting/dewetting on soft surfaces in-situ. The outcomes presented here may help as well in understanding the dynamics of evaporation and/or condensation of microdrops on such surfaces.

\section{Acknowledgements}

We thank Andreas Best for invaluable help with Laser Scanning Confocal Microscopy, Hans-Jürgen Butt for advice and fruitful discussions on the topic, and Mordechai 'Rudi' Sokuler for proof-reading. Sebastian Nett and Jochen Gutmann are acknowledged for assistance with the ink-jet printing technique.

\section{References}

1 P. G. de Gennes, Rev. Mod. Phys., 1985, 57, 827.

2 R. N. Wenzel, Ind. Eng. Chem., 1936, 28, 988.

3 A. B. D. Cassie, Discuss. Faraday Soc., 1948, 3, 11-16.

4 C. Huh and S. G. Mason, J. Colloid Interface Sci., 1977, 60, 11-38.

5 J. Drelich, J. D. Miller, A. Kumar and G. M. Whitesides, Colloids Surf., A, 1994, 93, 1-13.

6 A. Tuteja, W. Choi, M. Ma, J. M. Mabry, S. A. Mazzella, G. C. Rutledge, G. H. McKinley and R. E. Cohen, Science, 2007, 318, 1618-1622.

7 L. C. Gao and T. J. McCarthy, Langmuir, 2007, 23, 3762-3765.

8 G. R. Lester, J. Colloid Interface Sci., 1961, 16, 315-326.

9 A. I. Rusanov, Colloid J. USSR, 1975, 37, 614-622.

10 L. R. White, J. Colloid Interface Sci., 2003, 258, 82-96.

11 A. Carré, J. C. Gastel and M. E. R. Shanahan, Nature, 1996, 379, 432-434.

12 E. Saiz, A. P. Tomsia and R. M. Cannon, Acta Mater., 1998, 46, 2349-2361.

13 C. W. Extrand and Y. Kumagai, J. Colloid Interface Sci., 1996, 184, 191-200.

14 G. Pu and S. J. Severtson, Langmuir, 2008, 24, 4685-4692.

15 R. Pericet-Camara, A. Best, E. Bonaccurso and H.-J. Butt, Langmuir, 2008, 24, 10565-10568.

16 G. H. Fredrickson, A. Ajdari, L. Leibler and J. P. Carton, Macromolecules, 1992, 25, 2882-2889.

17 D. Long, A. Ajdari and L. Leibler, Langmuir, 1996, 12, 5221-5230.

18 Y.-S. Yu, Z. Yang and Y.-P. Zhao, J. Adhes. Sci. Technol., 2008, 22, 687-698.

19 M. E. R. Shanahan, J. Phys. D: Appl. Phys., 1988, 21, 981-985.

20 M. E. R. Shanahan and A. Carré, Langmuir, 1995, 11, 1396-1402.

21 M. Voué, R. Rioboo, C. Bauthier, J. Conti, M. Charlot and J. D. Coninck, J. Eur. Ceram. Soc., 2003, 23, 2769-2775.

22 J. G. Huddleston, A. E. Visser, W. M. Reichert, H. D. Willauer, G. A. Broker and R. D. Rogers, Green Chem., 2001, 3, 156-164.

23 S. Carda-Broch, A. Berthod and D. W. Armstrong, Anal. Bioanal. Chem., 2003, 375, 191-199.

24 T. W. Ridler and S. Calvard, IEEE Trans. Syst. Man Cybern., 1978, 8, 630-632.

25 T. Kawase, H. Sirringhaus, R. H. Friend and T. Shimoda, Adv. Mater., 2001, 13, 1601-1605.

26 E. Bonaccurso, H.-J. Butt, B. Hankeln, B. Niesenhaus and K. Graf, Appl. Phys. Lett., 2005, 86, 124101.

27 G. Li, K. Graf, E. Bonaccurso, D. S. Golovko, A. Best and H.-J. Butt, Macromol. Chem. Phys., 2007, 208, 2134-2144.

28 R. Pericet-Camara, A. Best, S. K. Nett, J. S. Gutmann and E. Bonaccurso, Opt. Express, 2007, 15, 9877-9882.

29 R. Pericet-Camara, E. Bonaccurso and K. Graf, ChemPhysChem, 2008, 9, 1738-1746.

30 V. V. Tsukruk, A. Sidorenko, V. V. Gorbunov and S. A. Chizhik, Langmuir, 2001, 17, 6715-6719.

31 B. Cappella and D. Silbernagl, Langmuir, 2007, 23, 10779-10787.

32 J. Domke and M. Radmacher, Langmuir, 1998, 14, 3320-3325. 